\title{
Cyclin-dependent kinase inhibitors exert distinct effects on patient-derived 2D and 3D glioblastoma cell culture models
}

\author{
Christin Riess ${ }^{1,2}$, Dirk Koczan ${ }^{3}$, Björn Schneider ${ }^{4}$, Charlotte Linke ${ }^{1}$, Katharina del Moral ${ }^{1}$, Carl Friedrich Classen ${ }^{1}$ and \\ Claudia Maletzki iD ${ }^{2}$
}

\begin{abstract}
Current therapeutic approaches have met limited clinical success for glioblastoma multiforme (GBM). Since GBM harbors genomic alterations in cyclin-dependent kinases (CDKs), targeting these structures with specific inhibitors (CDKis) is promising. Here, we describe the antitumoral potential of selective CDKi on low-passage GBM 2D- and 3D models, cultured as neurospheres (NSCs) or glioma stem-like cells (GSCs). By applying selective CDK4/6i abemaciclib and palbociclib, and the more global CDK1/2/5/9-i dinaciclib, different effects were seen. Abemaciclib and dinaciclib significantly affected viability in 2D- and 3D models with clearly visible changes in morphology. Palbociclib had weaker and cell line-specific effects. Motility and invasion were highly affected. Abemaciclib and dinaciclib additionally induced senescence. Also, mitochondrial dysfunction and generation of mitochondrial reactive oxygen species (ROS) were seen. While autophagy was predominantly visible after abemaciclib treatment, dinaciclib evoked $\mathrm{y}$-H2AX-positive double-strand breaks that were boosted by radiation. Notably, dual administration of dinaciclib and abemaciclib yielded synergistic effects in most cases, but the simultaneous combination with standard chemotherapeutic agent temozolomide (TMZ) was antagonistic. RNA-based microarray analysis showed that gene expression was significantly altered by dinaciclib: genes involved in cell-cycle regulation (different CDKs and their cyclins, SMC3), mitosis (PLK1, TTK), transcription regulation (IRX3, MEN1), cell migration/division (BCAR1), and E3 ubiquitination ligases (RBBP6, FBXO32) were downregulated, whereas upregulation was seen in genes mediating chemotaxis ( $C X C L 8, I L 6, C C L 2)$, and DNAdamage or stress (EGR1, ARC, GADD45A/B). In a long-term experiment, resistance development was seen in 1/5 cases treated with dinaciclib, but this could be prevented by abemaciclib. Vice versa, adding TMZ abrogated therapeutic effects of dinaciclib and growth was comparable to controls. With this comprehensive analysis, we confirm the therapeutic activity of selective CDKi in GBM. In addition to the careful selection of individual drugs, the timing of each combination partner needs to be considered to prevent resistance.
\end{abstract}

\section{Introduction}

Cyclin-dependent kinases (CDKs) play indispensable roles in a variety of biological processes, including cell-

\footnotetext{
Correspondence: Claudia Maletzki (claudia.maletzki@med.uni-rostock.de) ${ }^{1}$ University Children's Hospital, Rostock University Medical Centre, ErnstHeydemann-Straße 8, 18057 Rostock, Germany

2Department of Medicine Clinic III - Hematology, Oncology, Palliative Medicine, Rostock University Medical Center, Rostock University Medical Centre, ErnstHeydemann-Str. 6, 18057 Rostock, Germany

Full list of author information is available at the end of the article

Edited by Maria Victoria Niklison Chirou
}

cycle control, oncogenic transcription, DNA-damage repair, and stem cell self-renewal ${ }^{1,2}$. In most cancers, genomic alterations in specific CDKs either result in constitutive activation or loss of endogenous modulators, including those of the p16/CDK4-Cyclin-D/pRb pathway $^{3}$. This imbalance pushes cell-cycle progression and malignant transformation ${ }^{2}$. CDK inhibitors (CDKi's) specifically targeting these proteins are widely applied in (pre-)clinical oncological research ${ }^{1,4-8}$. CDKi has synergistic activity when applied in conjunction with other

\section{(c) The Author(s) 2021}

\footnotetext{
(c) (i) Open Access This article is licensed under a Creative Commons Attribution 4.0 International License, which permits use, sharing, adaptation, distribution and reproduction cc in any medium or format, as long as you give appropriate credit to the original author(s) and the source, provide a link to the Creative Commons license, and indicate if changes were made. The images or other third party material in this article are included in the article's Creative Commons license, unless indicated otherwise in a credit line to the material. If material is not included in the article's Creative Commons license and your intended use is not permitted by statutory regulation or exceeds the permitted use, you will need to obtain permission directly from the copyright holder. To view a copy of this license, visit http://creativecommons.org/licenses/by/4.0/.
} 
targeted drugs, such as BRAF and MEK inhibitors for malignant melanomas ${ }^{3}$. Most clinical trials have confirmed manageable toxicity profiles, with clinical responses in many cases and even significantly prolonged overall survival in selected patients cohorts ${ }^{9}$. To date, the three FDA-approved CDK4/6i abemaciclib, palbociclib, and ribociclib are a front-line treatment in combination with hormonal therapy for metastatic $\mathrm{HR}^{+}$-HER2 ${ }^{-}$breast cancer $(\mathrm{BC})^{9,10}$. Numerous ongoing clinical phase II and III studies evaluate the therapeutic potential in other entities. Functionally, CDK4/6 are cell-cycle-regulatory proteins that initiate the $\mathrm{G}_{1}-\mathrm{S}$-phase transition by interaction with $\mathrm{D}$-type cyclins and regulating $\mathrm{Rb}$ phosphorylation to activate or repress gene transcription ${ }^{2,11}$.

GBM is the most common and aggressive primary brain tumor ${ }^{12}$. Current therapeutic approaches using surgery and combined radio-/chemotherapy have met limited clinical success, contributing to the extremely poor 5 -year survival rate of $<3 \%{ }^{13,14}$. Genomic analysis revealed alterations in the $\mathrm{p} 16 / \mathrm{CDK} 4-\mathrm{Cyclin}-\mathrm{D} / \mathrm{pRb}$ pathway $^{13}$ as well as specific interphase CDKs, namely CDK1 and CDK5. The latter is strongly associated with tumor initiation ${ }^{15}$. As for GBM, a few studies have investigated the potential of CDKi's. Raub et al. described the antitumor activity of abemaciclib in an orthotopic glioblastoma rat model showing promising effects that were additive in combination with $\mathrm{TMZ}^{16}$. Another recent study even recommended nanoparticle encapsulated with dinaciclib in combination with radiation therapy for GBM via targeting tumor-associated macrophages ${ }^{17}$.

Here, we report the successful elimination of GBM cells by CDKi application with several morphology changes, including cell differentiation and vacuolization. We show that abemaciclib and the more global acting CDKi dinaciclib have individual effects on patient-derived 2D- and 3D models that result in senescence, autophagy, and mitochondrial impairment. By performing long-term in vitro treatment, developing resistance against dinaciclib can be prevented by abemaciclib, but not chemotherapy. These results are highly encouraging to move forward with this strategy.

\section{Results}

\section{CDKi treatment impairs viability in 2D- and 3D-cultured GBM cells}

In a preliminary pilot experiment, we applied the three CDKi's dinaciclib, abemaciclib, and palbociclib in monoand combination therapy with TMZ/radiation for $144 \mathrm{~h}$ using mostly clinically relevant doses. Abemaciclib is the only exception. Here, higher doses were applied.

As determined by light microscopy, several morphology changes were observed in GBM 2D cultures after dinaciclib and abemaciclib treatment. Exemplarily shown for
HROG05 and HROG63, dinaciclib treatment-induced small vacuoles and cell shrinkage. Abemaciclib-treated cells were enlarged, accompanied by a flattened structure and a striking multivacuolar phenotype (Fig. 1A). Notably, such morphological changes were seen in all cell lines and resulted in reduced cell viability (Fig. 1D). Palbociclib was less effective and TMZ treatment had no impact on viability and morphology at the doses used.

Then, we performed simultaneous and sequential combination regimens $\left(72 \mathrm{~h}\right.$ each, dose: $\left.\mathrm{IC}_{20}\right)$. The simultaneous treatment describes the concomitant administration of two substances, whereas the sequential regimen is characterized by consecutive administration of the respective drugs. In this comparative setting, only simultaneous, but not sequential treatment with dinaciclib and abemaciclib synergistically potentiated antitumor effects of the monotherapy in $3 / 5$ cases (Supplementary Table 1). With regard to TMZ, a synergistic effect was observed when this drug was added after dinaciclib, while the simultaneous treatment was mostly antagonistic. Combination of abemaciclib and TMZ showed also no benefit (Supplementary Table 1). Dual CDK blockade with dinaciclib and palbociclib was again only antagonistic (Supplementary Table 1).

In the 3D spheroids, cytotoxic effects of CDKi's were preserved. Still, we observed differences between individual 3D cultures, in which GSCs were more susceptible toward CDKi's than NSCs (Fig. 1B, C). In detail, dinaciclib and abemaciclib impaired GSC and NSC morphology, contributing to a significantly reduced viability (Fig. 1D). Here again, palbociclib had cell line-specific and only minor impact on viability. TMZ did not have any effect on 3D cultures (Fig. 1B, C). With regard to the combination, we again identified striking differences between simultaneous and sequential regimens and also between individual 3D cultures (Fig. 1D).

To sum up these findings, the timing of each combination partner influences effectiveness. Our results favor the sequential instead of the simultaneous treatment in both 2D- and 3D-cultured GBM cell lines. Also, palbociclib had lower activity against GBM cells than the other CDKi's dinaciclib and abemaciclib. Consequently, we focused on the latter two agents in further experiments.

\section{CDKi's induce apoptotic and necrotic cell death}

To describe the effects of CDKi's in more detail, we then performed flow cytometric apoptosis/necrosis analysis and focused on drug monoapplication. Figure 2 shows HROG63 as an example. Dinaciclib evoked necrosis, abemaciclib triggered early apoptosis (Fig. 2A, B). Dual CDK inhibition induces a mixed response but was not able to enhance cytotoxic effects (Fig. 2A, B). Immunogenic cell death, a common result of CDKi therapy, was not inducible by either treatment (Fig. 2C). 


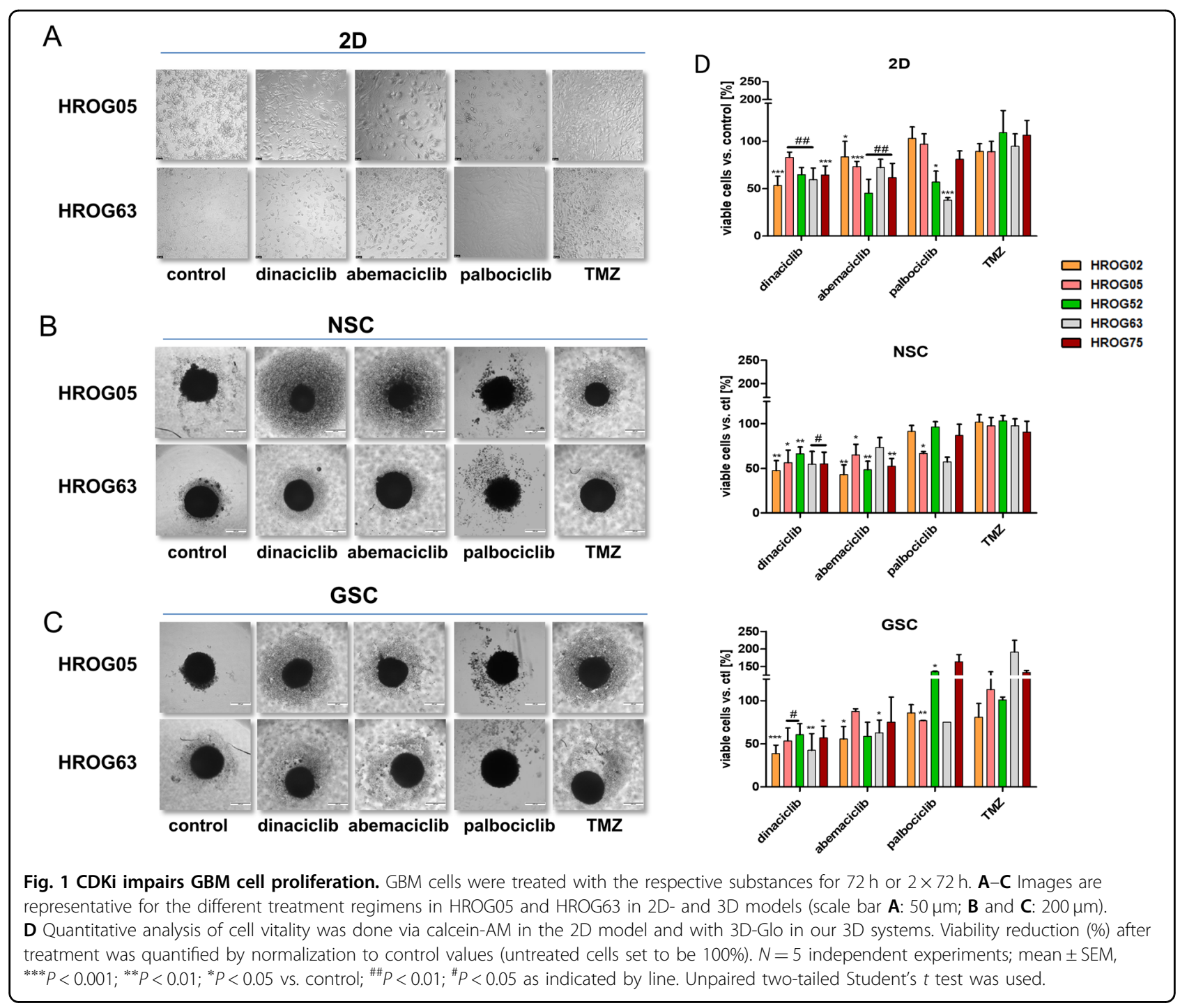

\section{CDKi's impair mitochondrial function and evoke methuosis-like processes}

Then, we analyzed the cause of cytoplasmic vacuole formation after CDKi treatment and screened the GBM cells for autophagy induction. Autophagy is responsible for maintaining cellular homeostasis, but promotes cell death after long-term stress. We indeed observed CDKiinduced lysosomal activation in HROG05 and HROG63, primarily in abemaciclib-treated cells (Fig. 3A). While this was hardly the only explanation for the observed morphology changes, we checked whether CDKi treatment causes mitochondrial damage. The mitochondrial membrane potential (MMP) increased in the 2D culture upon dinaciclib and abemaciclib, indicative of mitochondrial hyperpolarization (Fig. 3A). Such mitochondrial dysfunction may lead to oxidative stress and ROS production. Indeed, mono- and dual-CDKi administration additionally increased mitochondrial reactive oxygen species (mito-
ROS) levels (Fig. 3B), which corresponds to an increased MMP. TMZ alone or in combination with dinaciclib did not boost MMP and mito-ROS levels (Fig. 3A, B). Intensified MMP signals were partially accompanied by a higher intensity of the acidic activity. In HROG63 cells, MMP signals and acidic compartments overlapped, while this was not the case in HROG05 (Fig. 4A). Also, CDKiinduced huge vacuoles in HROG05 cells were neither positive for MMP, lysosomal activity, ER-Tracker, nor Dextran (Fig. 3C-E). Hence, CDKi may block the lysosomal processing of these vesicles.

So, we next investigated the origin of vacuole formation. By measuring LAMP-1/-2 and Rab7a via flow cytometry, single- and dual-CDKi application increased the percentage of LAMP1/2-Rab7a-positive cells. The cell line HROG05 showed the most pronounced morphological changes with massive vacuolization and LAMP1/2-Rab7a abundance under CDKi therapy (Fig. 3C-F). These 


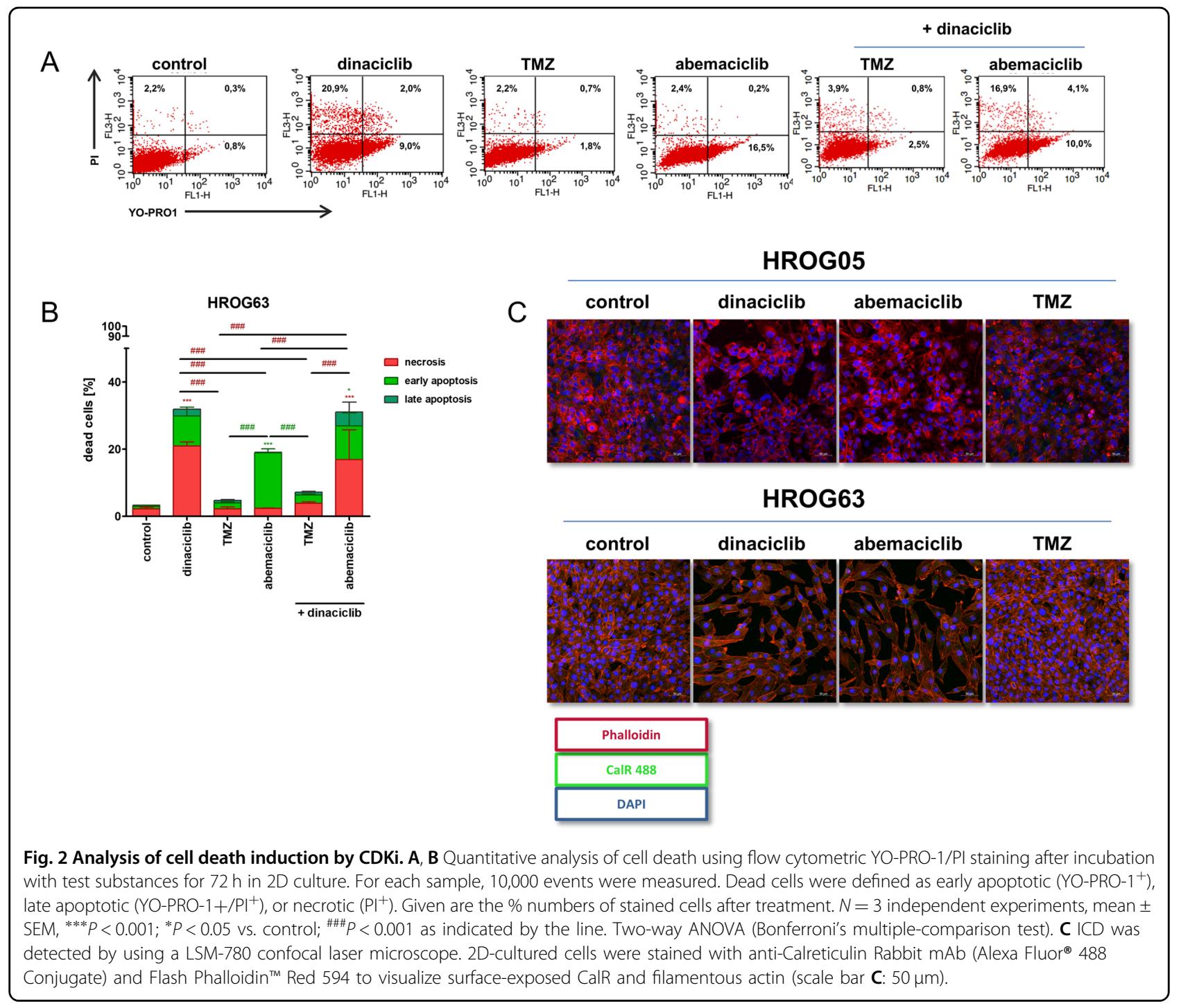

cumulative data are indicative of the induction of early methuosis-like processes.

To see whether similar mechanisms are present in 3D cultures, we next checked mitochondrial activity and hypoxia within NSC and GSC spheres (Fig. 3G). In both culture models, MMP was seen at the edge of the spheres. Mitochondrial hyperpolarization was especially seen after dinaciclib and abemaciclib treatment of NSC. Dual-CDKi treatment and dinaciclib in combination with TMZ accelerated the abundance of MMP signals, which were also visible in the inner sphere (Fig. 3G, upper part). Additional hypoxia analysis revealed that NSC had more hypoxic cores than GSC-irrespective of the applied treatment schedule (Fig. 3G, lower part). Mono- and dual-CDKi application increased hypoxia in these cells. In GSC, hypoxic cores increased after dinaciclib and abemaciclib monotherapy. The combination of CDKi's or with TMZ did not boost hypoxia significantly. Hence, hypoxia plays a minor role here.
Taken together, CDKi alone or in combination triggers a specific and uncommon mode of cell death that is characterized by a multivacuolar phenotype and signs of early methuosis.

\section{CDKi's trigger senescence induction in 2D- and 3D- cultured GBM cell lines}

Senescence can be triggered by different damaging stimuli, including telomere shortening (replicative senescence), oxidative DNA damage, and a persistent DNA damage response. Here, senescence is likely provoked by either ROS in hyperpolarized mitochondria (oxidative DNA damage) or accumulation of $\gamma \mathrm{H} 2 \mathrm{AX}$ foci that represent a subset of repair-proof lesions that seem to persist (DNA-damage response). Hence, we examined the activity of $\beta$-galactosidase, as well as activation of $\mathrm{p} 16 / \mathrm{p} 21$ in selected 2D- and 3D models as markers of senescence (Fig. 4). Senescence induction was observed in HROG05 and 


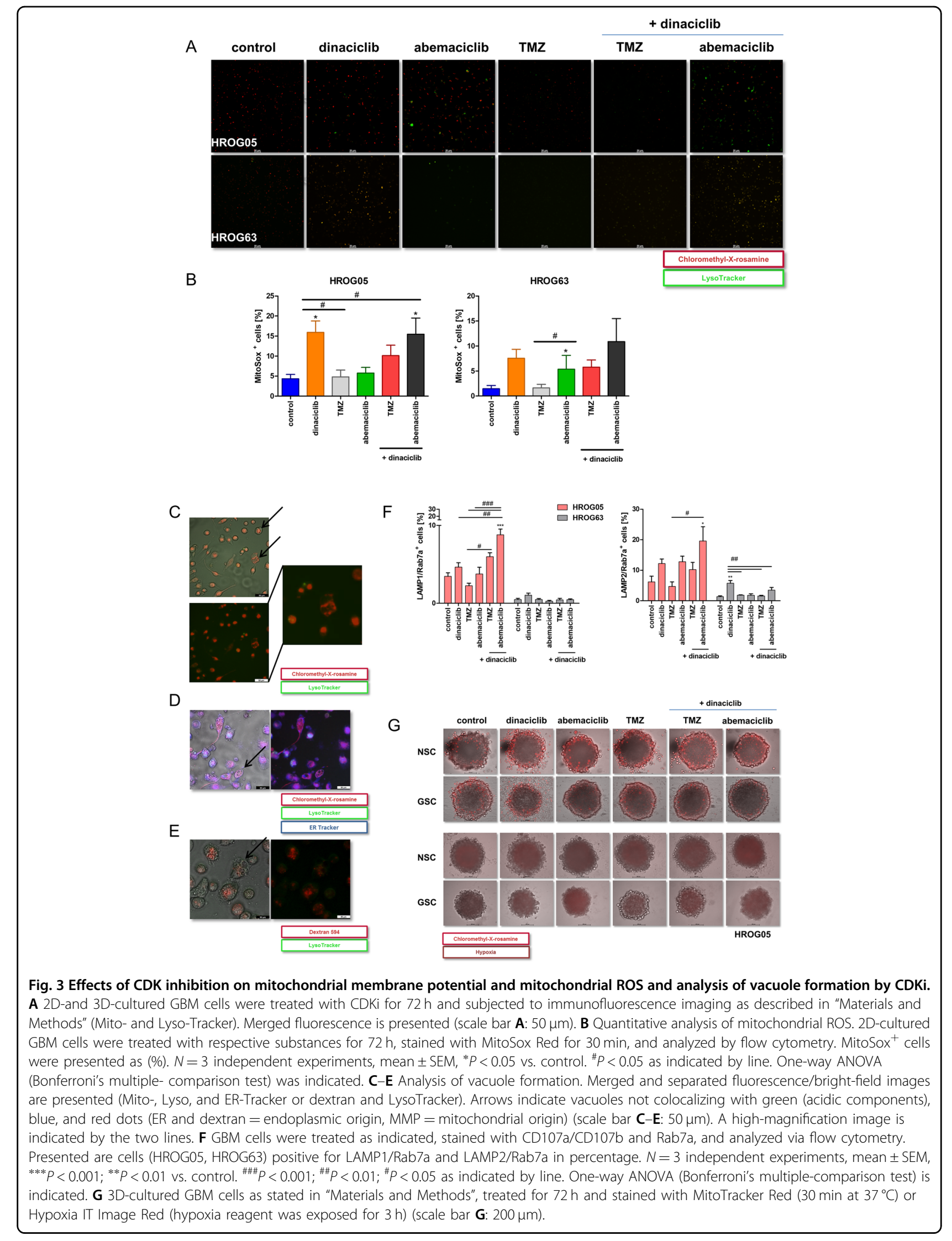




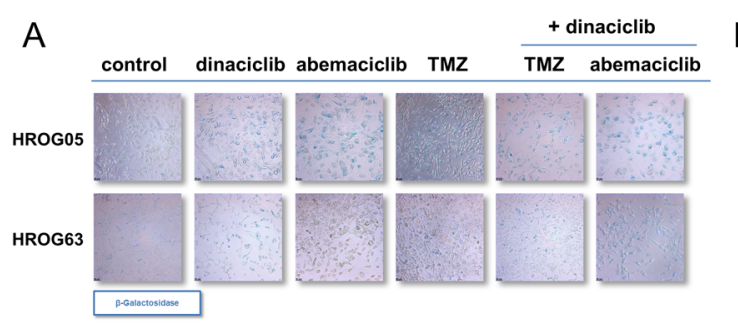

B
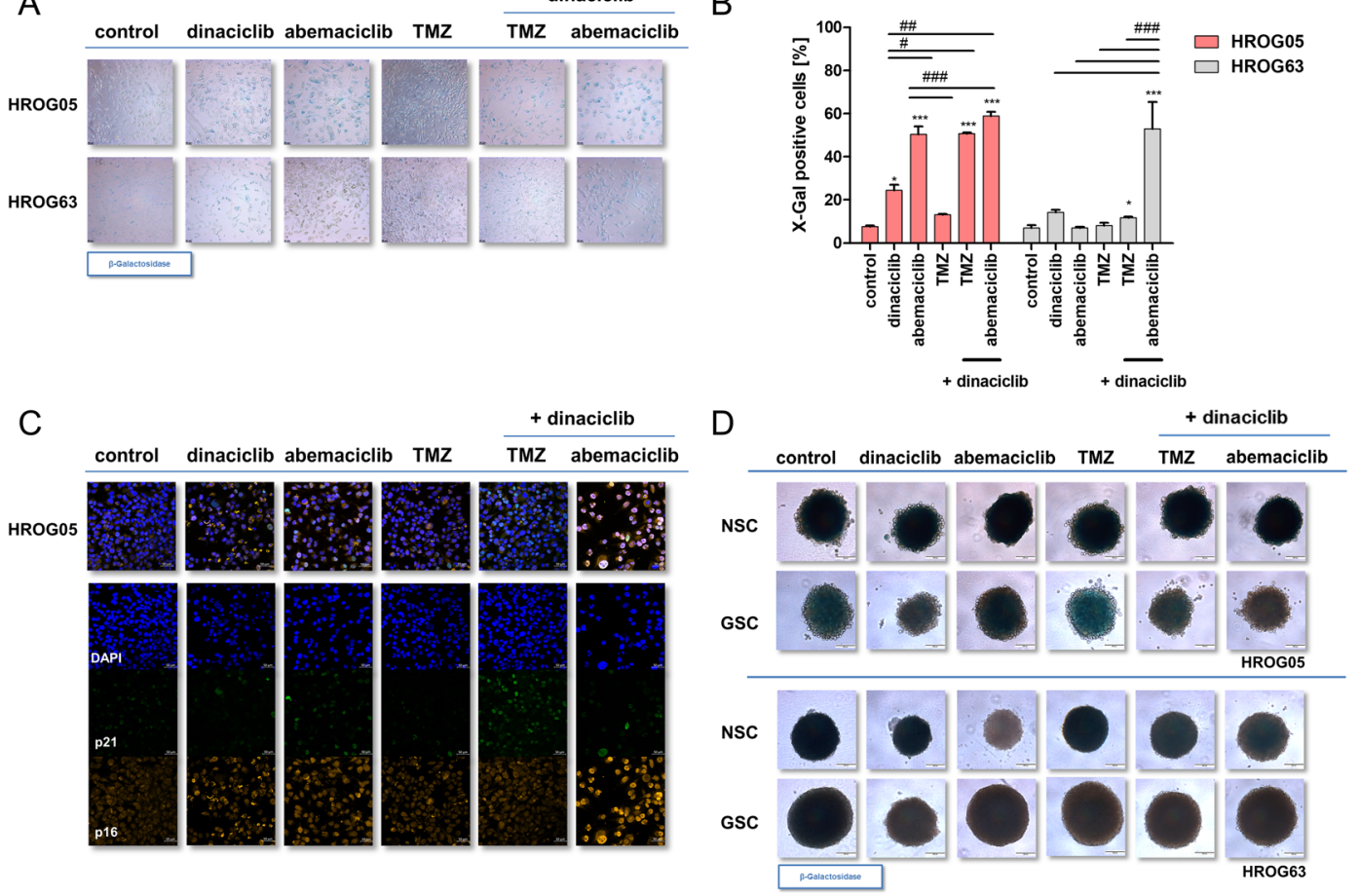

Fig. 4 CDK inhibition induces senescence via activation of p16/p21 and B-galactosidase. 2D- and 3D-cultured GBM cells (A, C, D) were treated with test substances for $72 \mathrm{~h}$, fixed, and stained with $\beta$-galactosidase staining solution overnight at $37^{\circ} \mathrm{C}$ without $\mathrm{CO}_{2}$. Blue: $\beta$-galactosidase activity, indicative for senescence. Representative images are shown (scale bar: A: $50 \mu \mathrm{m} ; \mathbf{C}: 200 \mu \mathrm{m}$ ). B Quantitative analyses of X-Gal-positive 2D-cultured cells in relation to the whole-cell number/image. $\mathbf{C}$ Representative images of GBM demonstrate an increase in p16 and p21 after CDK inhibition. The cell was treated as indicated, fixed, permeabilized, and stained with p21 Waf1/Cip1 (12D1) rabbit mAb (Alexa 488 conjugate) (green) and p16 antibody (JC8): sc-56330 Alexa 546 (orange) (scale bar: D: $20 \mu \mathrm{m}$ ). $N=3$ independent experiments, mean \pm SEM; ${ }^{* * *} P<0.001 ;{ }^{*} P<0.05$ vs. control;

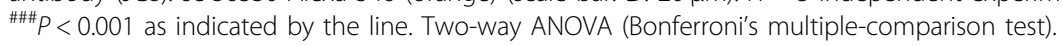

HROG63 cells, with, however, interindividual differences (Fig. 4A, B). Mono- and dual application of dinaciclib and abemaciclib was most effective. TMZ induced senescence in HROG05 cells, the combination with dinaciclib boosted the effects (Fig. 4A, B). The expression of senescence markers p16 and p21 increased in HROG05 cells after CDKi treatment and underlines the results of the $\beta$-galactosidase staining. p16 was detected in the nucleus and cytoplasm of the cells, whereas p21 was only found in the nucleus. Dual application of both CDKi leads to strong nuclear induction of p16. p21 expression was strongly elevated after dinaciclib and TMZ treatment. In some cases, e.g., in the combination regimens, colocalization of both markers was seen (Fig. 4C). Comparable, though less pronounced effects of $\beta$-galactosidase staining were observed in $3 \mathrm{D}$ cultures and again mainly visible in HROG05 cells (Fig. 4D). To sum up, CDKi's trigger senescence via activation of $\mathrm{p} 21$ and $\mathrm{p} 16$.

\section{CDKi's interfere with invasiveness and migratory potential of 2D- and 3D-cultured GBM cell lines}

In subsequent experiments, the impact of CDKi's treatment on cell motility as prerequisites for invasiveness and metastasis was studied in 2D- and 3D cultures.
First, a wound-healing assay was done. Figure 5A, B shows representative images of 2D-cultured HROG05 cells along with quantified scratch areas. Control and TMZ-treated cells proliferated appeared normal and almost closed the wound within 3 days (scratch completion after 7 days). Dinaciclib completely prevented GBM cell proliferation, which was characterized by cell shrinkage and cell death, leading to scratch areas higher than at day zero (Fig. 5A, B). Abemaciclib treatment decelerated wound healing and scratches did not fully close within the specified time of seven days (Fig. 5A, B). Dual CDKi treatment had comparable effects as the monotherapy; the addition of TMZ had no influence.

In a subsequent Matrigel-based invasion-migration assay, the migration and invasiveness of GBM cells slightly increased under dinaciclib treatment, likely constituting to some kind of escape. Abemaciclib reduced migration/invasion in HROG05 cells and led to a significant 4-fold decrease in HROG63 cells (Fig. 5C, $P<0.01$ vs. control).

To determine the effect of CDKi on GBM spheroids, we finally implanted defined individual NSC and GSC of cell line HROG05 in Matrigel and monitored sphere 

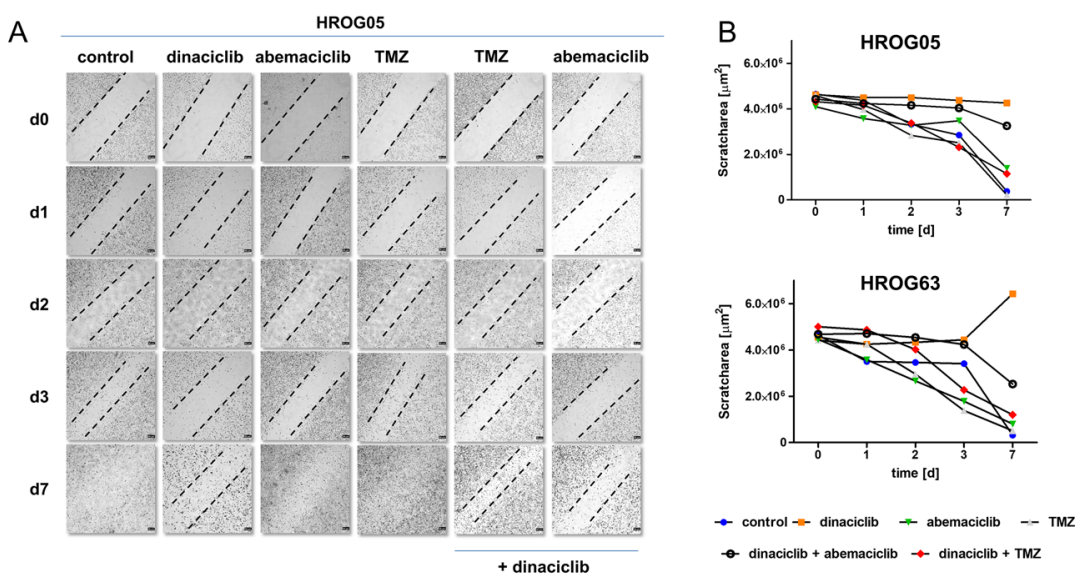

C

HROG63
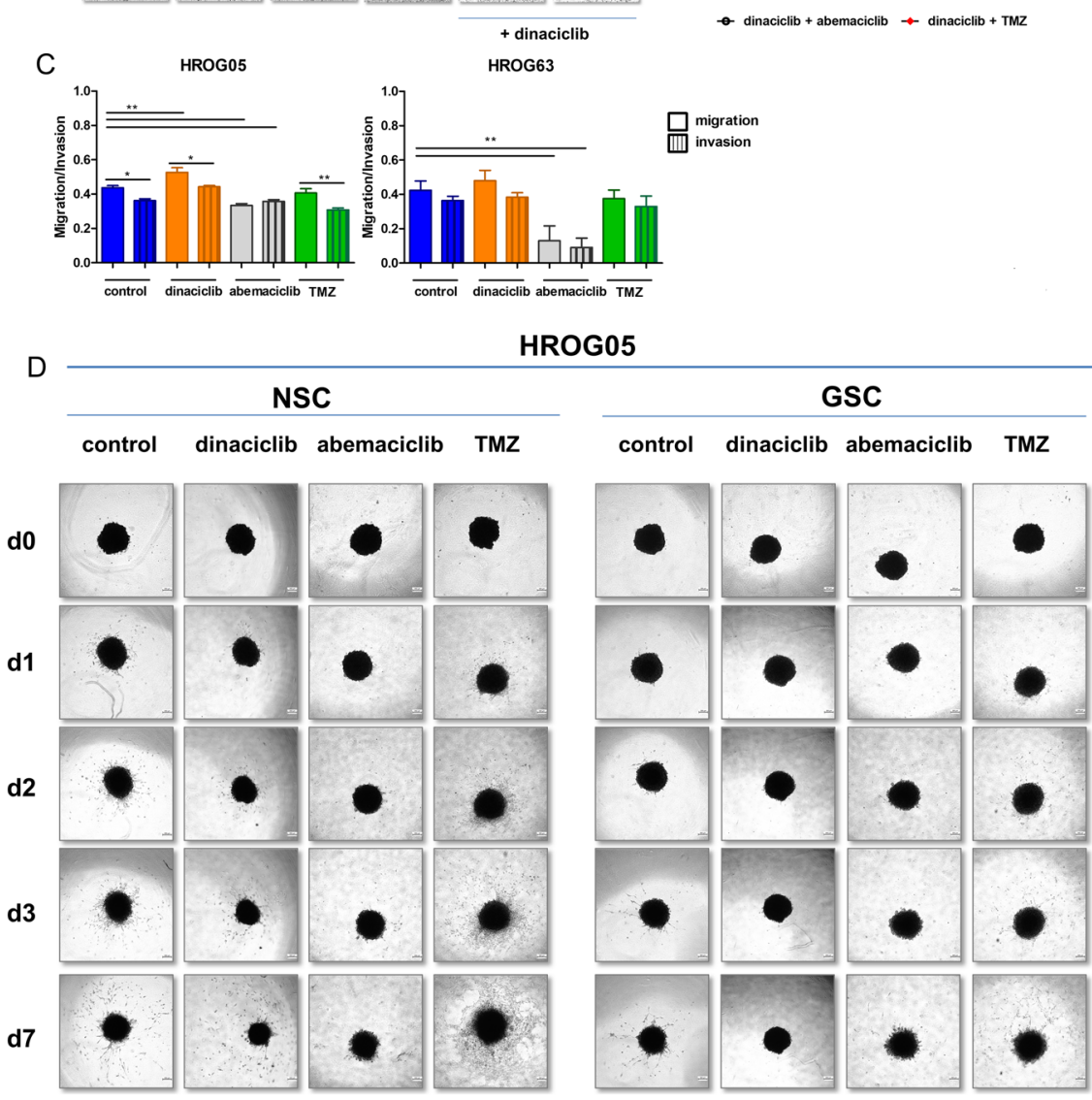

Fig. 5 The impact of CDKi on motility, migration, and invasion in 2D- and 3D models. A, B 2D-cultured GBM cells were seeded and grown to $90 \%$ confluency. A standard scratch was placed on the plate utilizing a $200-\mu l$ pipette tip. Detached cells were removed, and the above-mentioned substances were added. The scratch closure was monitored for 7 days using a Leica DMI 4000B microscope (scale bar A: $50 \mu \mathrm{m}$ ). For manual identification of scratch area (=open wound area), captured images were converted to grayscale. Finally, a threshold, a band-pass, and minimum filter were applied. Region of interest(s) (ROI) could then be set and scratch area analyzed. The scratch area $\left(\mu \mathrm{m}^{2}\right)$ was plotted over time for each substance. Data are presented as mean. C Effects of CDKi on cell invasion and migration assessed by a Boyden chamber assay. $N=3$ independent experiments, mean $\pm \mathrm{SEM},{ }^{* *} P<0.01 ;{ }^{*} P<0.05$ as indicated by the line. One-way ANOVA (Bonferroni's multiple-comparison test). D Analysis of CDKitreated 3D-cultured GBM cell invasion into a Matrigel matrix (scale bar D: $250 \mu \mathrm{m}$ ). GBM cells were monitored for a total of 7 days.

outgrowth (Fig. 5D). Control cells showed high basal invasiveness into the Matrigel in which NSCs were much more invasive than GSCs. CDKi treatment with dinaciclib or abemaciclib reduced their invasiveness, in some cases they did not even penetrate into the matrix (Fig. 5D).
However, TMZ treatment was not able to prevent invasiveness of spheroids, which was even higher than in the control. The main body of the implanted spheroid (NSC) was partially disintegrated due to the massive penetration into the matrix. 


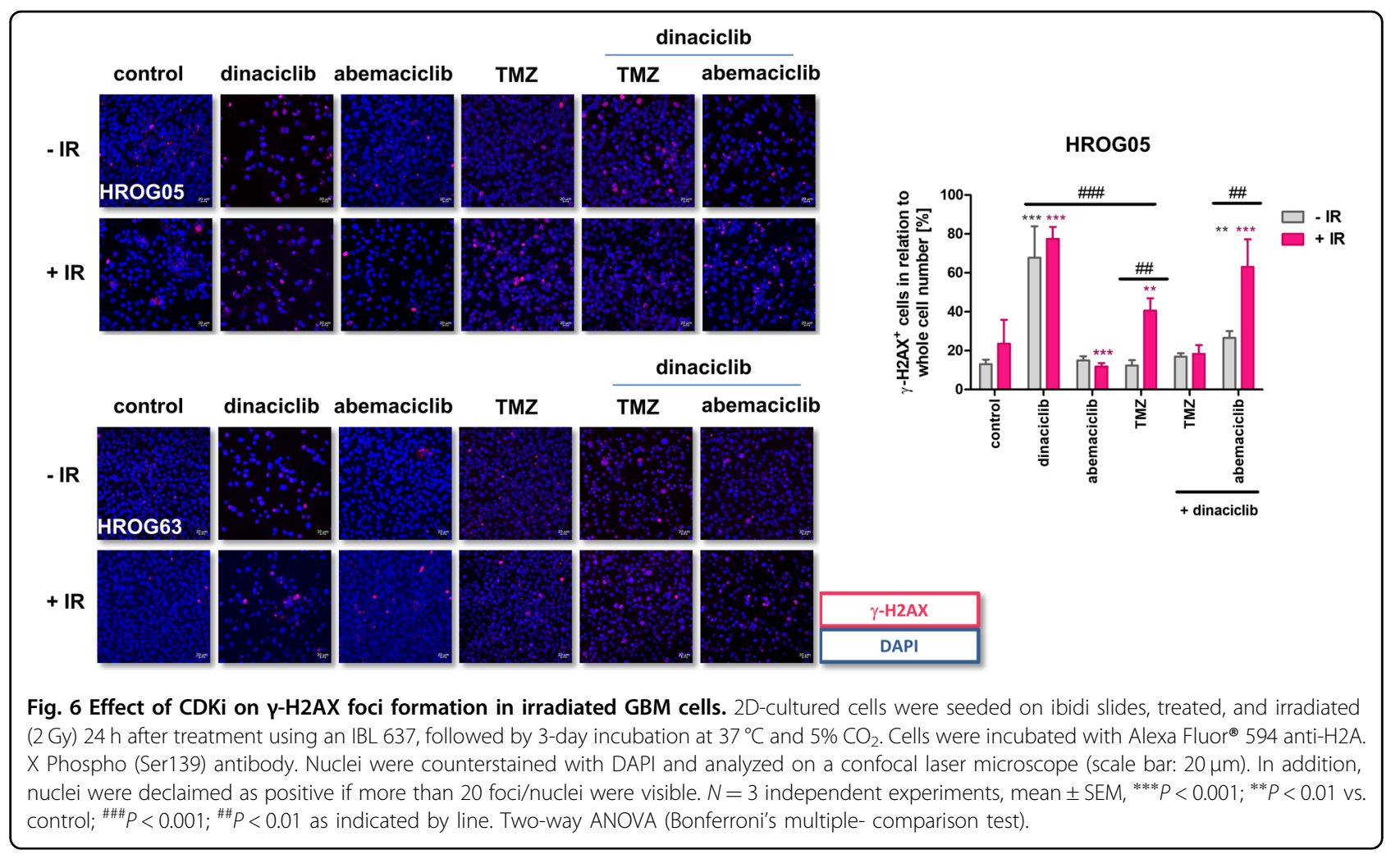

Hence, these findings nicely confirm the therapeutic potential of selective CDKi's to prevent invasion and migration. However, the addition of TMZ has a minor effect.

CDKi's have a minor impact on double-strand breaks and radiosensitivity

Thereafter, we evaluated the effects on signaling and DNA-damage repair. Double-strand breaks (DSB) were determined by $\gamma-\mathrm{H} 2 \mathrm{AX}$ staining, which promotes chromatin remodeling and the assembly of repair proteins (Fig. 6). Monotherapy of 2D-cultured cells with dinaciclib, but not abemaciclib elevated $\gamma-\mathrm{H} 2 \mathrm{AX}$ foci to a degree comparable to TMZ. Dual CDKi treatment or in combination with TMZ was not able to potentiate the effects of the monotherapy.

Then, we examined radiation-induced DSB. Radiation itself had little impact on $\gamma-\mathrm{H} 2 \mathrm{AX}$ foci, but dinaciclib pretreatment boosted DSB. No such radiosensitizing effect was seen for abemaciclib. TMZ likewise induced radiation-induced DSB, but in combination with dinaciclib, this effect completely vanished in HROG05 cells whereas this was not the case in HROG63 cells.

Microarray analysis identifies molecular alterations upon dinaciclib and confirms the therapeutic activity

As a part of our study, we conducted microarray analyses of 2D-cultured HROG63 cells, either treated with dinaciclib or left untreated (Fig. 7 and Supplementary Table 2). Among the 8008 genes in this analysis having a fold change of 2 and a $P$ value of $<0.05,4447$ were up- and 3561 downregulated.

The most downregulated genes were BCAR1, PLK1, TTK, SIN3A, CENPF, and SMC3, all being involved in cell-cycle and mitosis inhibition. Genes mediating transcription regulation (RBBP6, IRX3, RTF1, and MEN1), chromosome remodeling (HIST1H1B), and those encoding for E3 ubiquitination ligases (RBBP6, FBXO32, CUL1) were also strongly downregulated. The expression levels of CXCL8, ARC, EGR1, TFPI2, GADD45B, CCL20, EGR1, PTGS2, JUNB, FOS, CCL2, DUSP2, IER5, and IL6 mRNA significantly increased. CDKs that were downregulated involved the known targets CDK1 (CCNA2, B1/2) and CDK9 (CCNK, T1) as well as other CDKs, including CDK7 (CCNA2, B1/2, E1), CDK12 (CCNK), CDK17, and CDK20. While senescence was already confirmed by SA$\beta$-Gal staining, senescence-associated genes (SERPINB2/ $E 1, B T G 2$, and NEK4) were also elevated. The same applies to genes regulating autophagy and apoptosis. The former were upregulated ( $U L K 2, M A P 1 L C 3 A / B, B E C N 1$, ATG12, DRAM2, SQSTM1, and EI24), the latter (CASP genes) showed mostly downregulation. CASP5 is the only exception.

Taken together, these molecular data nicely underpin our findings on the complex effects of the multi-CDKi dinaciclib on GBM cells. 


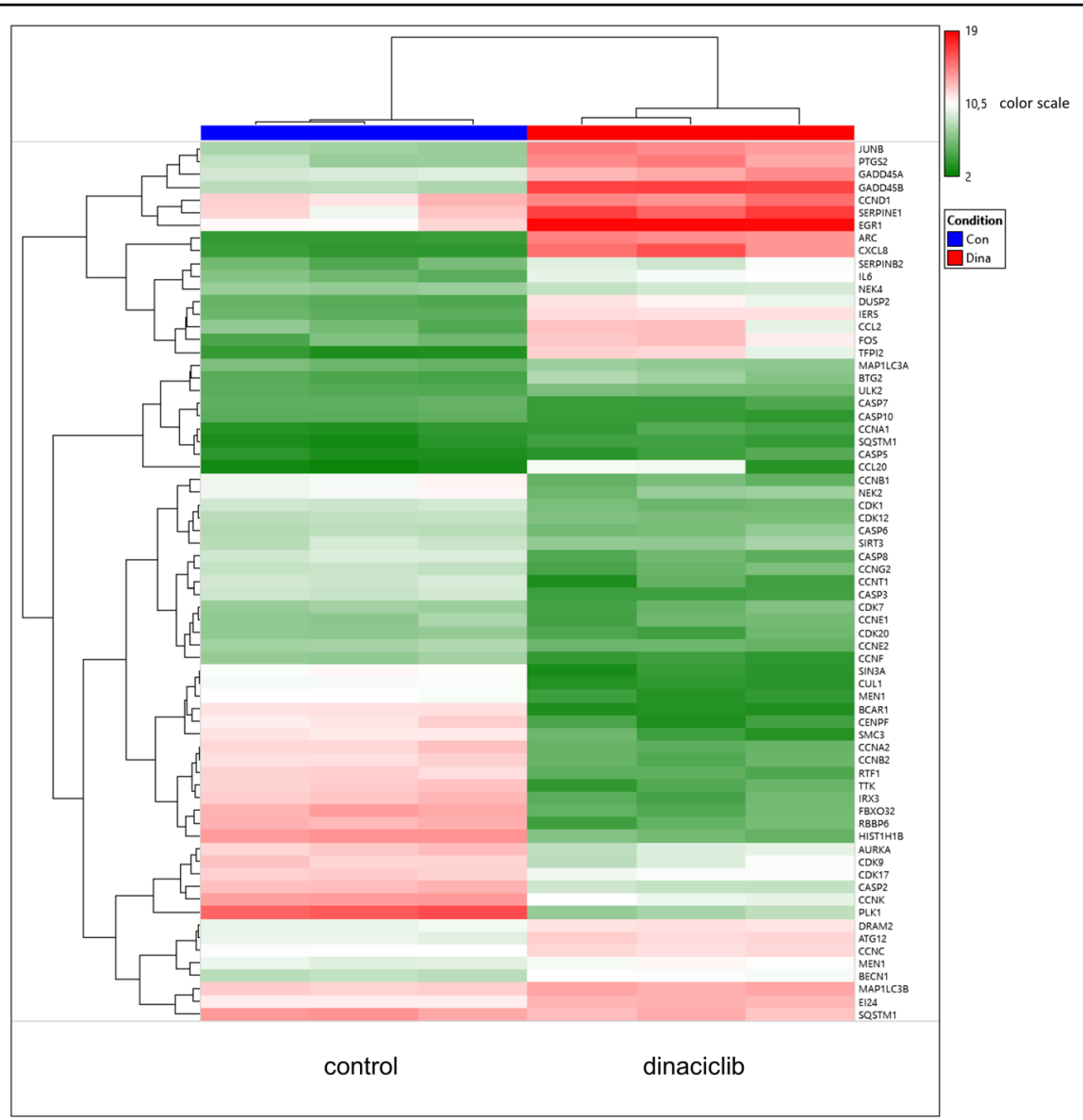

Fig. 7 Molecular alterations upon dinaciclib. Heatmap showing RNA expression level from 2D-cultured HROG63 cells assessed by Affymetrix Human Clariom S Array. Primary data analysis was performed with the Affymetrix TAC including the SST-RMA for normalization. Gene expression data were log-transformed. Limma was used here to calculate the $p$-value. A change was considered significant when the Limma eBayes $P$ value met the criterion $P<0.05$ at fold changes $>|2|$, i.e., expression increments or declines larger than two. $N=3$ independent experiments.

\section{Resistance development can be abrogated by combined CDK inhibition}

Finally, we studied resistance development under ongoing treatment. A long-term treatment approach of ten repetitive weekly cycles was carried out on 2Dcultured cells. Crystal-violet and calcein-AM/MitoTracker staining were used to visualize effects (Fig. 8).

Dinaciclib long-term treatment completely inhibited colony formation in $3 / 5$ cases. Interestingly, most colonies were seen in HROG05 cells that already displayed the weakest sensitivity toward dinaciclib in 2D short-term therapy. Hence, is it likely that a pre-existing resistant clone was responsible for outgrowth. The other cell line was HROG63, showing small colonies after dinaciclib long-term exposure. Notably, no colony formation was seen after repetitive abemaciclib treatment (Fig. 8). Hence, we formally confirm the therapeutic activity of this CDKi. As anticipated and in addition to the minor effects after short-term exposure, TMZ did not influence colony formation, with growth comparable to controls. Adding dinaciclib to TMZ prevented colony formation in one case (HROG52), but abrogated the therapeutic effect in the remaining cell lines (Fig. 8). The combination of both CDKi was effective in four cases; still, continued growth was seen in cell line HROG63. It is therefore tempting to speculate that the same clone seen with long-term dinaciclib monotherapy was responsible for colony formation in this setting.

Summing up, these data provide evidence that intrinsic rather than acquired resistance plays a role in CDKi treatment failure.

\section{Discussion}

In this study, we provide functional evidence for the antitumoral effects of mono- and dual treatment with CDKi on low-passage GBM models and identify mechanisms of response. Notably, all patient-derived GBM cell lines tested in this study were sensitive to abemaciclib and dinaciclib, while the overall response to palbociclib was weaker and additionally cell line-specific. 


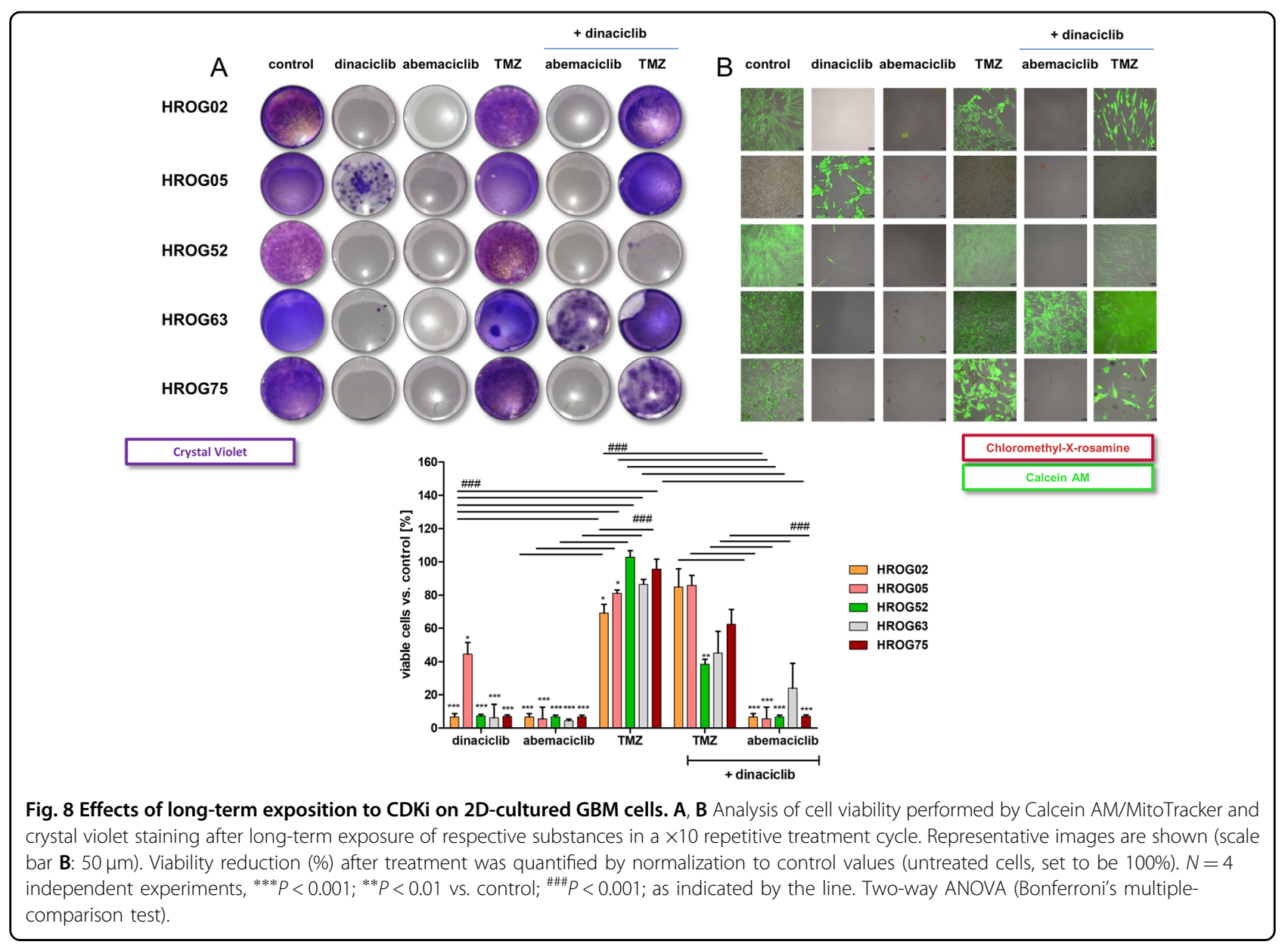

Abemaciclib is structurally distinct from palbociclib, with higher selectivity for CDK4 than CDK6 and targeting additional kinases, including GSK3 $\alpha / \beta$ and CAMKII $\alpha / \beta /$ $\gamma^{18}$, which may explain the individual effects of the two CDK4/6i seen here. In support of this, we also observed different responses to the CDK4/6i in the 3D-spheroid system. By including two individual 3D-culture models (NSC, GSC) that closely resemble in vivo features, these data favor prospective abemaciclib and dinaciclib application instead of palbociclib in treating GBM patientsalso due to the blood-brain-barrier permeability of abemaciclib. To underpin this, the mode of cell death was studied in more detail. Here, we focused on abemaciclib and dinaciclib. While both agents induced autophagy with different intensities, abemaciclib additionally increased early apoptotic cells, while dinaciclib predominantly evoked necrosis. CDKi-mediated apoptosis has been described in various tumor models ${ }^{19-23}$. By contrast, autophagy was only recently described for abemaciclib ${ }^{24}$ and just one publication described the cytoprotective role of autophagy under dinaciclib therapy in NSCLC ${ }^{25}$. Though not analyzed in detail here, LC3B-II aggregation and decreased p62/sequestosome1 expression levels are likely alterations as earlier shown for flavopiridol, another pan-CDKi ${ }^{26}$. In addition to the effects described above, abemaciclib and dinaciclib induced senescence via activation of p16/p21 and likewise evoked DNA-damage response, also confirmed by changes in mRNA expression pattern.

Cell motility and invasion are prerequisites for tumor progression and metastasis. Here, abemaciclib decelerated motility and migration/invasion of GBM cells. Dinaciclib completely prevented GBM motility, but was unable to affect migration/invasion in the 2D system. Other studies already described reduced motility in solid tumors under CDKi treatment. CDK4 inhibition was shown to decrease invasion, metastatic spread, and tumor progression in a RB-high pancreatic ductal adenocarcinoma model ${ }^{27}$. One report even suggested the involvement of CDK5 in the metastatic spread; another trial proposed the contribution of vimentin, Snail, COX-2, and PGE2 $2^{27-30}$. Strikingly, both CDKi prevented invasiveness of NSC and GSC spheroids. Although the natural invasive behavior was higher in NSC compared to GSC, TMZ equally enhanced invasive growth in both 3D models. In NSC, the main body of the implanted spheroid was partially disintegrated 
into the matrix. In addition to CDK4 and CDK5, cyclin-B/ CDK1 may play a role in tumor cell spreading, motility, and invasion ${ }^{31,32}$. Previous studies have also shown that inhibition of CDK2/9 in triple-negative BC cells and CDK9 inhibition in osteosarcoma cells decreased migration by preventing phosphorylation of CDK-mediated Smad3 and RNA POL-ll in triple-negative $\mathrm{BC}^{33,34}$.

We further demonstrated mitochondrial dysfunction characterized by elevated MMP levels and overproduction of mito-ROS. The results from the 2D system were additionally confirmed in 3D models showing elevated MMP upon dinaciclib. While the inner sphere showed less MMP signals, they were visible on the edge of the sphere. Mono- and dual CDKi increased hypoxia in NSC, indicating loss of mitochondrial function mainly in the inner sphere ${ }^{35,36}$.

We, therefore, hypothesize that mono- or dual CDKi treatment increases oxidative stress, induces DSB, and potentiates senescence to trigger cell death ${ }^{37-42}$. While MMP signals and acidic compartments overlapped in 2Dcultured HROG63 cells, this was not the case in cell line HROG05. Intriguingly, we also observed multiple huge vacuoles in CDKi-treated cells. CDKi seemed to trigger the uptake of $10-\mathrm{kDa}$ dextrans and the acidotropic agent in small vacuoles, but rarely in the most enlarged ones. Also, huge vacuoles were insensitive for ER-Tracker and MMP, suggesting vacuoles also did not originate from the mitochondria or endoplasmic reticulum. Here, vacuolization was accompanied by a higher abundance of LAMP1/2 and Rab7a, which are late endosomal markers formed in the early stages of methuosis. Methuosis is a nonapoptotic cell death phenotype defined as the accumulation of large fluid-filled cytoplasmic vacuoles that originate from macropinosomes ${ }^{43}$. Here, these characteristics superficially may resemble methuosis(-like) processes in addition to the appearance of apoptosis ${ }^{43}$. Recent data from the literature support this mechanism, as some antineoplastic agents, including abemaciclib promote vacuolization, which may lead to methuosis $^{24,44,45}$.

GBM cells may have intrinsic and/or acquired radioresistance. Dinaciclib, but not abemaciclib induced $\gamma$ H2AX foci that were boosted after combined CDKi radiotherapy. The induction of DSBs was accompanied by downregulation of $C D K 1$ and SIRT3, the latter being known to mediate radioresistance ${ }^{46}$. Hence, dinaciclib reversed radioresistance.

By directly comparing the cytotoxic activity of abemaciclib and dinaciclib, the latter was more potent, likely due to the global activity in targeting multiple CDK. Finally, the strong cytotoxic effects of dinaciclib were confirmed in the microarray data of HROG63 cells. Genes involved in cell-cycle regulation/progression, mitosis, transcription regulation, cell migration/adhesion/division, and those encoding for E3 ubiquitination ligases were strongly downregulated, whereas the expression level of chemotaxis-mediating and DNA-damage or stress genes was significantly upregulated. The upregulation of DDR genes such as $G A D D 45 A / B$ which affects aurora-A and Nek2 and therefore promotes genomic instability and histone alterations ${ }^{47,48}$ along with reduced $C D K 1$ and SIRT3 expression may explain the treatment-induced accumulation of $\gamma-\mathrm{H} 2 \mathrm{AX}$. While CDKs are not only involved in cell-cycle progression, they play also crucial roles in neuronal differentiation, transcription regulation, and migration/invasion. Here, several CDKs and their corresponding cyclins are downregulated. Among them, $C D K 1$ is required for successful completion of $\mathrm{M}$-phase but also contributes to DNA-damage repair, checkpoint activation, and the progression of senescence escape by modulating the survivin pathway in glioma cells ${ }^{49,50}$. Though dinaciclib is widely described as CDK $1 / 2 / 5 / 9 \mathrm{i}$, we also detected downregulation of genes encoding for $C D K 9, C D K 12$, and $C D K 20$ which may have an impact on the protein level. CDK20 promotes cell growth and facilitates radio-chemoresistance in lung cancer cells ${ }^{51}$. CDK12, a transcriptional regulator of homologous recombination that shares sequence homology with CDK9, was previously identified as a target of dinaciclib in $\mathrm{BC}$ cells. The therapeutic effect included resensitization to a PARP inhibitor yielding durable regression in a patientderived xenograft model ${ }^{52}$. Dinaciclib has an even more complex mode of action than previously anticipated. This not only involves cell-cycle arrest, but also cell death via numerous mechanisms: impaired DNA-damage repair, genomic instability, disturbed transcription regulation, and induction of dysregulated mitochondria, senescence, and autophagy. Whether abemaciclib likewise alters gene expression on such a global level, is a matter of speculation and has to be addressed prospectively. Still, our complex set of data indicate that similar mechanisms are altered by abemaciclib. Quite in line, long-term treatment with abemaciclib prevented colony formation even better than dinaciclib. Small viable colonies in some cases might be best explained by single outgrowing clones either intrinsically resistant or rapidly acquiring resistance upon therapeutic pressure. Residual cells showed MMP signals, with reduced viability, indicative of nonintact or almost dying cells.

Summarizing our findings, we show that abemaciclib and dinaciclib, but not palbociclib, inhibited GBM viability/ motility, and invasion through multiple mechanisms: senescence, autophagy, necrosis/apoptosis, and mitochondrial dysfunction. We additionally provide mechanistic insights regarding the single-agent activity of dinaciclib. Our data support the idea of using CDKs as therapeutic targets in GBM and suggest dual CDKi application as a new therapeutic approach in clinical trials. 


\section{Materials and methods}

Patient-derived tumor cell lines and culture conditions

Patient-derived GBM cell lines $(N=5$; HROG02, HROG05, HROG52, HROG63, HROG75) were established in our lab and basically characterized, including molecular analysis and MGMT promoter methylation status $^{53}$. 2D cell cultures were cultured in full medium and incubated at $37^{\circ} \mathrm{C}$ in a humidified atmosphere of $5 \%$ $\mathrm{CO}_{2}$ : Dulbecco's modified eagle medium: nutrient mixture F-12 supplemented with 10\% FCS, L-glutamine (2mmol/l), and antibiotics $(100 \mathrm{U} / \mathrm{ml}$ penicillin $/ 100 \mu \mathrm{g} /$ $\mathrm{ml}$ streptomycin) (all from Pan Biotech, Aidenbach, Germany). 3D GBM neurosphere cells (NSC) and glioma stem-like cells (GSC) were cultured in ultra-lowattachment (ULA) plates (Greiner Bio-One, Kremsmünster, Austria) in a defined medium. NSC was grown in serum-containing medium (=sphere medium), whereas GSC was cultivated in serum-free medium containing stem cell-inducing additives ( $=$ stem cell medium) $(+1 \times$ B-27 ${ }^{\circledR}$ supplement $(50 \times)\left(\right.$ Gibco $^{\mathrm{TM}}$, Life Technologies ${ }^{\mathrm{TM}}$, Carlsbad, USA), $+20 \mathrm{ng} / \mathrm{ml}$ recombinant human epidermal growth factor (rhEGF), +10 ng/ml bFGF (Immunotools, Friesoythe, Germany). Prior treatment, cells were incubated until spheroids form $(\sim 72-96 \mathrm{~h})$. Using this methodology, a single well-defined spheroid with cell line-specific appearance of a particular size was generated.

\section{Cytostatic drugs and targeted substance}

The cytostatics included the CDKi's (all from Selleckchem, Munich, Germany) abemaciclib $(10 \mu \mathrm{M})$, palbociclib $(10 \mu \mathrm{M})$, and dinaciclib (10 or $100 \mathrm{nM})$. TMZ $(10 \mu \mathrm{M})$ was obtained from MSD (Haar, Germany). All substances were used in doses below the $\mathrm{IC}_{50}$ as determined in the preliminary dose-finding study (range $10 \mathrm{nM}-10 \mu \mathrm{M})$.

\section{Viability and senescence assays}

Cell viability was assessed by calcein-acetoxymethyl (AM) (Biomol GmbH, Hamburg, Germany) fluorometric assay in $2 \mathrm{D}$ culture as described in ref. ${ }^{54}$. 3D cultures were analyzed luminometric using the CellTiter-Glo ${ }^{\circledR} 3 \mathrm{D}$ cell viability assay (Promega, Walldorf, Germany) according to the manufacturer's instruction. CellTiterGlo ${ }^{\circledR} 3 \mathrm{D}$ luminescence signal was read with a microplate reader (Infinite ${ }^{\circledR}$ M200, Tecan Group, Switzerland). In addition, ten cycles of single and combined therapy were done in long-term treatment. Readout was done by Calcein AM + MitoTracker Red (Cell Signaling Technology, Frankfurt/Main, Germany) and crystal violet staining (0.2\%, Sigma-Aldrich, St. Louis, USA). Senescenceassociated $\beta$-galactosidase (SA- $\beta$-gal, Cell Signaling Technology, Cambridge, UK), as well as apoptosis and necrosis using flow cytometry-based Yo-Pro1/propidium iodide staining, was measured as described in refs. ${ }^{54,55}$. For p16 and p21 detection, cells were fixed with $2 \%$ paraformaldehyde (PFA) w/o methanol (15 min, Thermo Fisher Scientific, Darmstadt, Germany), washed twice, permeabilized, and blocked with $2 \%$ BSA, $0.5 \%$ Triton X100 in PBS for $60 \mathrm{~min}$ and stained with p21 Waf1/Cip1 (12D1) rabbit mAb (Alexa 488 Conjugate) (green) (1:300, Cell Signaling Technology) and p16 antibody (JC8): sc56330 Alexa 546 (orange) (1:50, Santa Cruz Biotechnology, Dallas, TX) overnight at $4{ }^{\circ} \mathrm{C}$. Nuclei were counterstained with DAPI and cells were analyzed on a Zeiss LSM-780 Confocal Laser Microscope (Zeiss, Jena, Germany).

\section{Immunogenic cell death assays}

GBM cells were treated for $72 \mathrm{~h}$. Cells were fixed in $4 \%$ PFA w/o methanol (30 min)). Surface-exposed Calreticulin (CalR) was detected using an anti-Calreticulin Rabbit mAb (Alexa Fluor ${ }^{\circledR} 488$ conjugate) (1:50; overnight, Cell Signaling Technology), followed by filamentous actin staining (Flash Phalloidin ${ }^{\mathrm{TM}}$ Red 594 (1:20, 20 min, Biolegend). Nuclei were counterstained with DAPI and cells analyzed on a Zeiss LSM-780 confocal laser microscope.

\section{Cs-137 $\mathrm{\gamma}$-irradiation}

Cs-137 $\gamma$-irradiation (2Gy) was performed $24 \mathrm{~h}$ after treatment using an IBL 637 (CIS Bio-International, Codolet, France), followed by 3-day incubation at $37{ }^{\circ} \mathrm{C}$ and $5 \% \mathrm{CO}_{2}$. Double-strand breaks (DSB) were assessed with $\gamma-\mathrm{H} 2 \mathrm{AX}$ staining. For the latter, cells were fixed with $4 \%$ PFA/PBS, washed twice, permeabilized $(0.5 \%$ Triton $\mathrm{X}-100,15 \mathrm{~min}$ ), blocked in 1\% BSA (45 min), and incubated with Alexa Fluor ${ }^{\circledR} 594$ anti-H2A.X Phospho (Ser139) antibody (1:1000, overnight, $4{ }^{\circ} \mathrm{C}$ ) (Biolegend). Nuclei were counterstained with DAPI and analyzed on a Zeiss LSM-780 confocal laser microscope.

\section{In vitro wound-healing assay and 2D cellular migration/ invasion assay}

A wound-healing assay was done as described in ref. ${ }^{56}$, data acquisition was made using a Leica DMI 4000B microscope (Leica, Heidelberg, Germany). Then, a modified Boyden chamber technique (ThinCerts, Greiner Bio-One) with and without Matrigel-coated membranes (Corning, Corning, USA) was applied according to Ramer et al. ${ }^{57}$.

\section{Tumor spheroid invasion assay}

After sphere formation, 96-ULA well plates were placed on ice; half of the medium was removed, and reagents were added at a twofold final concentration $(+\mathrm{EGF}=$ to stimulate invasion) into U-bottom wells containing icecold matrigel (Corning). Spheres were monitored for 7 days and images were taken at $24 \mathrm{~h}$ to 3 -day intervals with a final record on day 7 (Leica DMI 4000B). 


\section{MitoTracker ${ }^{\circledR}$ Red CMXRos, MitoSOX ${ }^{\mathrm{TM}}$ Red, LysoTracker ${ }^{\mathrm{TM}}$ Green DND-26 ER Tracker ${ }^{\mathrm{TM}}$ Blue-White DPX, and Dextran Alexa Fluor $^{\mathrm{TM}}$ 594; 10,000 MW}

MitoTracker CMXRos (8-(4'-chloromethyl) phenyl2,3,5,6,11,12,14,15-octahydro-1 H,4 H,10 H,13 H-diquinolizino-8 H-xanthylium chloride), LysoTracker Green, ERTracker $^{\mathrm{TM}}$ Blue-White (Dapoxyl) DPX and Molecular Probes $^{\mathrm{TM}}$ Dextran, Alexa Fluor ${ }^{\mathrm{TM}}$ 594; 10,000 MW, anionic, fixable dyes were prepared according to the manufacturer's instructions (Cell Signaling Technology, Thermo Fisher Scientific). Mitochondria (MitoTracker Red CMXRos, $20 \mathrm{nM}$ ), ER (ER Tracker, $500 \mathrm{nM}$ ), and acidic components (LysoTracker, $50 \mathrm{nM}$ ) were stained $\left(30 \mathrm{~min}, 37^{\circ} \mathrm{C}\right) 72 \mathrm{~h}$ post treatment; Dextran $(100 \mu \mathrm{g} / \mathrm{ml})$ was given simultaneously with treatment and incubated $72 \mathrm{~h}$. Images were taken on fluorescence microscopy (Leica DMI 4000B). MitoSOX ${ }^{\mathrm{TM}} \operatorname{Red}\left(5 \mu \mathrm{M}, 30 \mathrm{~min}, 37^{\circ} \mathrm{C}\right.$, Thermo Fisher Scientific) was used as indicator of mitochondrial superoxide and analyzed via flow cytometry (FACS Verse, BD Bioscience, Franklin Lakes, USA).

\section{LAMP1/2, Rab7a measurement}

Vacuole formation was quantified on cells stained with CD107a, CD107b (lysosomal-associated membrane proteins 1 and 2, LAMP1, LAMP2), and GTPase Ras-related protein (Rab7a; $30 \mathrm{~min}, 4{ }^{\circ} \mathrm{C}$, + intracellular staining) (all purchased from Biolegend). The analysis was done on a FACS Verse.

\section{Image-iT hypoxia reagent}

$3 \mathrm{D}$ cultures were maintained, plated, and treated as described. Hypoxia reagent (Thermo Fisher Scientific) was added $\left(10 \mu \mathrm{M}, 3 \mathrm{~h}, 37^{\circ} \mathrm{C}\right)$. The medium was replaced and cells kept in the incubator $(24 \mathrm{~h})$. Cells were imaged using a fluorescence microscope (Leica DMI 4000B).

\section{Microarray analysis of RNA expression profiles}

RNA of treated and control HROG63 cells $\left(5 \times 10^{5}\right.$ cells/treatment) were extracted employing RNeasy Plus Kit (Qiagen, Hilden, Germany) according to the manufacturer's protocol. The total RNA was quantified on a spectrophotometer (NanoDrop 1000, Thermo Fisher Scientific) and integrity confirmed using the Agilent Bioanalyzer 2100 with an RNA Nano chip kit (both from Agilent Technologies, Waldbronn, Germany). Expression profiling was performed by taking advantage of the Affymetrix Human Clariom S Array (Affymetrix/Thermo Fisher Scientific, Santa Clara, USA), which interrogates over 20,000 well-annotated genes. Therefore, the so-called Whole Transcriptome protocol was employed described in ref. ${ }^{58}$. Primary data analysis was performed with the Affymetrix Transcriptome Analysis Console (TAC) software, including the SST-RMA for normalization. Gene expression data were log-transformed. Limma was used here to calculate the $P$ value. A change was considered significant when the Limma eBayes $P$ value met the criterion $P<0.05$ at fold changes $>|2|$, i.e., expression increments or declines larger than two.

\section{Image processing}

Quantification of the images was done by using the FIJIImageJ software as follows:

Staining intensity was determined by dividing the channels into red, green, and blue. Subsequently, integrated density profiles of the same size were measured in the respective channels.

For manual identification of scratch area (= open wound area), captured images were converted to grayscale. Finally, a threshold, a band-pass, and minimum filter were applied. Region of interest(s) (ROI) could then be set and scratch area analyzed. The scratch area $\left[\mu \mathrm{m}^{2}\right]$ was plotted over the time for each substance. Data are presented as mean.

\section{Statistics}

All values are given as mean \pm SEM. After proving the assumption of normality, differences between controls and treated cells were determined by using the unpaired Student's $t$-test viability determination. In the case of multiple comparisons, one- or two-way ANOVA on ranks (Bonferroni's multiple-comparison test) was applied. Statistical evaluation was performed using GraphPad PRISM software, version 5.02 (GraphPad Software, San Diego, CA, USA). The criterion for significance was taken to be $P<0.05$.

\section{Acknowledgements}

Open Access funding enabled and organized by Projekt DEAL.

\begin{abstract}
Author details
'University Children's Hospital, Rostock University Medical Centre, ErnstHeydemann-Straße 8, 18057 Rostock, Germany. ${ }^{2}$ Department of Medicine Clinic III - Hematology, Oncology, Palliative Medicine, Rostock University Medical Center, Rostock University Medical Centre, Ernst-Heydemann-Str. 6, 18057 Rostock, Germany. ${ }^{3}$ Core Facility for Microarray Analysis, Institute for Immunology, Rostock University Medical Centre, 18057 Rostock, Germany. ${ }^{4}$ Institute of Pathology, Strempelstraße 14, 18055 Rostock, Rostock University Medical Centre, 18057 Rostock, Germany
\end{abstract}

\section{Author contributions}

C.R., D.K., and K.D.M. performed all experiments, D.K. performed the microarray, C.L. did the p21/p16 immunofluorescence staining, K.D.M. the Boydenchamber assay, C.M. and C.R. designed the study, analyzed, and interpreted the data, prepared all figures, and performed statistical analysis; C.R. and C.M. wrote the paper, all authors participated in paper finalization and critically revised the paper.

\section{Funding}

This work was supported by a grant from the Lieselotte Beutel Stiftung to C. Riess and C. Maletzki. 


\section{Conflict of interest}

The authors declare that the research was conducted in the absence of any commercial or financial relationships that could be construed as a potential conflict of interest.

\section{Publisher's note}

Springer Nature remains neutral with regard to jurisdictional claims in published maps and institutional affiliations.

Supplementary information The online version contains supplementary material available at https://doi.org/10.1038/s41420-021-00423-1.

Received: 29 September 2020 Revised: 23 November 2020 Accepted: 23 December 2020

Published online: 15 March 2021

\section{References}

1. Sherr, C. J., Beach, D. \& Shapiro, G. I. Targeting CDK4 and CDK6: from discovery to therapy. Cancer Discov. 6, 353-367 (2016)

2. Sánchez-Martínez, C., Lallena, M. J., Sanfeliciano, S. G. \& de Dios, A. Cyclin dependent kinase (CDK) inhibitors as anticancer drugs: recent advances (2015-2019). Bioorg. Med. Chem. Lett. 29, 126637 (2019).

3. Martin, C. A. et al. Palbociclib synergizes with BRAF and MEK inhibitors in treatment naïve melanoma but not after the development of BRAF inhibitor resistance. Int. J. Cancer 142, 2139-2152 (2018).

4. Syn, N. L. et al. Pan-CDK inhibition augments cisplatin lethality in nasopharyngeal carcinoma cell lines and xenograft models. Signal Transduct. Target Ther. 3, 9 (2018)

5. Schaer, D. A. et al. The CDK4/6 inhibitor abemaciclib induces a T cell inflamed tumor microenvironment and enhances the efficacy of PD-L1 checkpoint blockade. Cell Rep. 22, 2809-2817 (2018).

6. Parry, D. et al. Dinaciclib (SCH 727965), a novel and potent cyclin-dependent kinase inhibitor. Mol. Cancer Ther. 9, 2344-2353 (2010).

7. Li, C., Qi, L., Bellail, A. C., Hao, C. \& Liu, T. PD-0332991 induces G1 arrest of colorectal carcinoma cells through inhibition of the cyclin-dependent kinase-6 and retinoblastoma protein axis. Oncol. Lett. 7, 1673-1678 (2014).

8. Hsu, C.-L. et al. Integrated genomic analyses in PDX model reveal a cyclindependent kinase inhibitor Palbociclib as a novel candidate drug for nasopharyngeal carcinoma. J. Exp. Clin. Cancer Res. 37, 233 (2018).

9. Sobhani, N. et al. Updates on the CDK4/6 inhibitory strategy and combinations in breast cancer. Cells 8, 321 (2019).

10. Schoninger, S. F. \& Blain, S. W. The ongoing search for biomarkers of CDK4/6 inhibitor responsiveness in breast cancer. Mol. Cancer Ther. 19, 3-12 (2020).

11. Canepa, E. T. et al. INK4 proteins, a family of mammalian CDK inhibitors with novel biological functions. IUBMB Life. 59, 419-426 (2007).

12. Silantyev, A. S. et al. Current and future trends on diagnosis and prognosis of glioblastoma: from molecular biology to proteomics. Cells 8, 863 (2019).

13. Solomon, D. A., Kim, J.-S., Jean, W. \& Waldman, T. Conspirators in a capital crime: co-deletion of p18INK4c and p16INK4a/p14ARF/p15INK4b in glioblastoma multiforme. Cancer Res. 68, 8657-8660 (2008).

14. Lah, T. T, Novak, M. \& Breznik, B. Brain malignancies: glioblastoma and brain metastases. Semin Cancer Biol. 60, 262-273 (2020).

15. Mandl, M. M. et al. Inhibition of Cdk5 induces cell death of tumor-initiating cells. Br. J. Cancer 116, 912-922 (2017).

16. Raub, T. J. et al. Brain exposure of two selective dual CDK4 and CDK6 inhibitors and the antitumor activity of CDK4 and CDK6 inhibition in combination with temozolomide in an intracranial glioblastoma xenograft. Drug Metab. Dispos. 43, 1360-1371 (2015)

17. Zhang, P. et al. Therapeutic targeting of tumor-associated myeloid cells synergizes with radiation therapy for glioblastoma. Proc. Natl Acad. Sci. USA 116, 23714-23723 (2019).

18. Wu, T. et al. Effect of abemaciclib (LY2835219) on enhancement of chemotherapeutic agents in $A B C B 1$ and $A B C G 2$ overexpressing cells in vitro and in vivo. Biochem Pharmacol. 124, 29-42 (2017).

19. Torres-Guzmán, R. et al. Preclinical characterization of abemaciclib in hormone receptor positive breast cancer. Oncotarget 8, 69493-69507 (2017).

20. Zhao, H. et al. Study of the mechanism by which dinaciclib induces apoptosis and cell cycle arrest of lymphoma Raji cells through a CDK1-involved pathway. Cancer Med. 8, 4348-4358 (2019).
21. Alsayegh, K, Matsuura, K., Sekine, H. \& Shimizu, T. Dinaciclib potently suppresses MCL-1 and selectively induces the cell death in human iPS cells without affecting the viability of cardiac tissue. Sci. Rep. 7, 1-13 (2017).

22. Jane, E. P. et al. Dinaciclib, a cyclin-dependent kinase inhibitor promotes proteasomal degradation of $\mathrm{Mcl}-1$ and enhances ABT-737-mediated cell death in malignant human glioma cell liness. J. Pharm. Exp. Ther. 356, 354-365 (2016).

23. Tanaka, Y. et al. Abemaciclib, a CDK4/6 inhibitor, exerts preclinical activity against aggressive germinal center-derived B-cell lymphomas. Cancer Sci. 111, 749-759 (2020).

24. Hino, $\mathrm{H}$. et al. Abemaciclib induces atypical cell death in cancer cells characterized by formation of cytoplasmic vacuoles derived from lysosomes. Cancer Sci. 111, 2132-2145 (2020).

25. Jeong, E. H. et al. Anti-tumor effect of CDK inhibitors on CDKN2A-defective squamous cell lung cancer cells. Cell Oncol. 41, 663-675 (2018).

26. Wang, S., Wang, K., Wang, H., Han, J. \& Sun, H. Autophagy is essential for flavopiridol-induced cytotoxicity against MCF-7 breast cancer cells. Mol. Med Rep. 16, 9715-9720 (2017)

27. Chou, A. et al. Tailored first-line and second-line CDK4-targeting treatment combinations in mouse models of pancreatic cancer. Gut 67, 2142-2155 (2018).

28. Feldmann, G. et al. Cyclin-dependent kinase inhibitor Dinaciclib (SCH727965) inhibits pancreatic cancer growth and progression in murine xenograft models. Cancer Biol. Ther. 12, 598-609 (2011).

29. Bisht, S. et al. Cyclin-dependent kinase 5 (CDK5) controls melanoma cell motility, invasiveness, and metastatic spread-identification of a promising novel therapeutic target. Transl. Oncol. 8, 295-307 (2015).

30. Qin, G. et al. Palbociclib inhibits epithelial-mesenchymal transition and metastasis in breast cancer via c-Jun/COX-2 signaling pathway. Oncotarget 6, 41794-41808 (2015).

31. Singh, M. K. et al. HEl10 negatively regulates cell invasion by inhibiting cyclin B/Cdk1 and other promotility proteins. Oncogene 26, 4825-4832 (2007).

32. Fang, L., Du, W. W., Awan, F. M., Dong, J. \& Yang, B. B. The circular RNA circCcnb1 dissociates Ccnb1/Cdk1 complex suppressing cell invasion and tumorigenesis. Cancer Lett. 459, 216-226 (2019).

33. Ma, H., Seebacher, N. A., Hornicek, F. J. \& Duan, Z. Cyclin-dependent kinase 9 (CDK9) is a novel prognostic marker and therapeutic target in osteosarcoma. EBioMedicine 39, 182-193 (2019).

34. Thomas, A. L. et al. Inhibition of CDK-mediated Smad3 phosphorylation reduces the Pin1-Smad3 interaction and aggressiveness of triple negative breast cancer cells. Cell Cycle 16, 1453-1464 (2017).

35. Baracca, A., Sgarbi, G., Solaini, G. \& Lenaz, G. Rhodamine 123 as a probe of mitochondrial membrane potential: evaluation of proton flux through FO during ATP synthesis. Biochim Biophys. Acta - Bioenerg. 1606, 137-146 (2003).

36. Solaini, G., Baracca, A., Lenaz, G. \& Sgarbi, G. Hypoxia and mitochondrial oxidative metabolism. Biochim. Biophys. Acta - Bioenerg. 1797, 1171-1177 (2010).

37. Cui, H., Kong, Y. \& Zhang, H. Oxidative stress, mitochondrial dysfunction, and aging. J. Signal Transduct. 2012, 1-13 (2012).

38. Zorova, L. D. et al. Functional significance of the mitochondrial membrane potential. Biochem. Suppl. Ser. A Membr. Cell Biol. 12, 20-26 (2018).

39. Zorova, L. D. et al. Mitochondrial membrane potential. Anal. Biochem. $\mathbf{5 5 2}$ 50-59 (2018).

40. Brand, M. D. Mitochondrial generation of superoxide and hydrogen peroxide as the source of mitochondrial redox signaling. Free Radic. Biol. Med. 100 14-31 (2016).

41. Venkatachalam, G., Surana, U. \& Clément, M. V. Replication stress-induced endogenous DNA damage drives cellular senescence induced by a sub-lethal oxidative stress. Nucleic Acids Res. 45, 10564-40582 (2017).

42. Coluzzi, E., Leone, S. \& Sgura, A. Oxidative stress induces telomere dysfunction and senescence by replication fork arrest. Cells 8, 19 (2019).

43. Maltese, W. A. \& Overmeyer, J. H. Methuosis: nonapoptotic cell death associated with vacuolization of macropinosome and endosome compartments. Am. J. Pathol. 184, 1630-1642 (2014)

44. Silva-Pavez, E. et al. CK2 inhibition with silmitasertib promotes methuosis-like cell death associated to catastrophic massive vacuolization of colorectal cancer cells. Cell Death Dis. 10, 73 (2019).

45. Overmeyer, J. H., Young, A. M., Bhanot, H. \& Maltese, W. A. A chalcone-related small molecule that induces methuosis, a novel form of non-apoptotic cell death, in glioblastoma cells. Mol. Cancer 10, 69 (2011).

46. Liu, R. et al. CDK1-mediated SIRT3 activation enhances mitochondrial function and tumor radioresistance. Mol. Cancer Ther. 14, 2090-2102 (2015). 
47. Salvador, J. M., Brown-Clay, J. D. \& Fornace, A. J. Gadd45 in stress signaling, cell cycle control, and apoptosis. Adv. Exp. Med Biol. 793, 1-19 (2013).

48. Nikonova, A. S., Astsaturov, I., Serebriiskii, I. G., Dunbrack, R. L. \& Golemis, E. A. Aurora A kinase (AURKA) in normal and pathological cell division. Cell. Mol. Life Sci. 70, 661-687 (2013).

49. Liao, H. et al. CDK1 promotes nascent DNA synthesis and induces resistance of cancer cells to DNA-damaging therapeutic agents. Oncotarget 8, 90662-90673 (2017).

50. Song, Z. et al. Escape of U251 glioma cells from temozolomide-induced senescence was modulated by CDK1/survivin signaling. Am. J. Transl. Res. 9 2163-2180 (2017)

51. Wang, Q. et al. CDK20 interacts with KEAP1 to activate NRF2 and promotes radiochemoresistance in lung cancer cells. Oncogene 36, 5321-5330 (2017).

52. Johnson, S. F. et al. CDK12 inhibition reverses de novo and acquired PARP inhibitor resistance in BRCA wild-type and mutated models of triple-negative breast cancer. Cell Rep. 17, 2367-2381 (2016).
53. Mullins, C. S. et al. Establishment and characterization of primary glioblastoma cell lines from fresh and frozen material: a detailed comparison. PLOS ONE 8 , e71070 (2013).

54. Maletzki, C. et al. Host defense peptides for treatment of colorectal carcinomaa comparative in vitro and in vivo analysis. Oncotarget 5, 4467-4479 (2014).

55. Maletzki, $\mathrm{C}$. et al. Deciphering molecular mechanisms of arginine deiminasebased therapy - comparative response analysis in paired human primary and recurrent glioblastomas. Chem. Biol. Interact. 278, 179-188 (2017).

56. Oehmcke-Hecht, S. et al. Streptococcus gallolyticus abrogates anticarcinogenic properties of tannic acid on low-passage colorectal carcinomas. Sci. Rep. 10, 1-10 (2020).

57. Ramer, R., Rohde, A., Merkord, J., Rohde, H. \& Hinz, B. Decrease of plasminogen activator inhibitor-1 may contribute to the anti-invasive action of cannabidiol on human lung cancer cells. Pharm. Res. 27, 2162-2174 (2010).

58. Koczan, D., Fitzner, B., Zettl, U. K. \& Hecker, M. Microarray data of transcriptome shifts in blood cell subsets during S1P receptor modulator therapy. Sci. Data 5, 180145 (2018). 